\title{
SIR distribution analysis in cellular networks considering the joint impact of path-loss, shadowing and fast fading
}

\author{
Dorra Ben Cheikh ${ }^{1,2}$, Jean-Marc Kelif ${ }^{1}$, Marceau Coupechoux ${ }^{2^{*}}$ and Philippe Godlewski ${ }^{2}$
}

\begin{abstract}
In this paper, we propose an analysis of the joint impact of path-loss, shadowing and fast fading on cellular networks. Two analytical methods are developed to express the outage probability. The first one based on the Fenton-Wilkinson approach, approximates a sum of log-normal random variables by a log-normal random variable and approximates fast fading coefficients in interference terms by their average value. We denote it FWBM for Fenton-Wilkinson based method. The second one is based on the central limit theorem for causal functions. It allows to approximate a sum of positive random variables by a Gamma distribution. We denote it CLCFM for central limit theorem for causal functions method. Each method allows to establish a simple and easily computable outage probability formula, which jointly takes into account path-loss, shadowing and fast fading. We compute the outage probability, for mobile stations located at any distance from their serving BS, by using a fluid model network that considers the cellular network as a continuum of BS. We validate our approach by comparing all results to extensive Monte Carlo simulations performed in a traditional hexagonal network and we provide the limits of the two methods in terms of system parameters. The proposed framework is a powerful tool to study performances of cellular networks, e.g., OFDMA systems (WiMAX, LTE).
\end{abstract}

\section{Introduction}

In this paper, we are interested in characterizing the signal to interference ratio (SIR) distribution on the downlink of a cellular network. The SIR outage probability and the SIR cumulative distribution function (CDF) are important metrics for the performance evaluation of wireless communication systems. In this paper, we define the outage probability as the probability that the SIR at the input of the receiver chain is falling below a given threshold value. This performance parameter is crucial for both coverage and capacity studies. In terms of coverage, mobile stations should be able to decode common control channels (like pilots or broadcast channels) and thus to attain a certain SIR threshold on these channels with high probability. In this case, we are interested in the low SIR region of the SIR distribution in order to evaluate the cell coverage. In terms of capacity and for systems implementing link adaptation on

\footnotetext{
* Correspondence: marceau.coupechoux@telecom-paristech.fr

${ }^{2}$ TELECOM ParisTech \& CNRS LTCI, Paris, France

Full list of author information is available at the end of the article
}

shared downlink channels (such as HSPA or LTE), the whole SIR distribution is needed for performance evaluation. The ergodic capacity at a certain distance from the base station is indeed evaluated as an expectation of the Shannon classical formula over the channel variations. The cell capacity is obtained by integration over the cell area.

The issue of expressing outage probability in cellular networks has been extensively addressed in the literature. For this study, a difficult task is to take into account the joint impact of path-loss, shadowing and fast fading. There are two classical assumptions: (1) considering only the shadowing effect, (2) considering both shadowing and fast fading effects. In the former case, authors mainly face the problem of expressing the distribution of the sum of log-normally random variables; several classical methods can be applied to solve this issue (see e.g. $[1,2])$. In the latter case, formulas usually consist in many infinite integrals, which are uneasy to handle in a practical way (see e.g. [3]). In both cases, outage probability is always an explicit function of all distances from the user to interferers. 
Table 1 CDF difference in dB between Monte Carlo simulations (SIM) on the one hand and CLCFM and FWBM on the other hand at 5, 50 and $90 \%(\sigma=3,4,6$ $\mathrm{dB}$, * means greater than $3 \mathrm{~dB}$ ).

\begin{tabular}{|c|c|c|c|c|}
\hline$\sigma(\mathrm{dB})$ & $\eta$ & $r[\mathrm{~km}]$ & $\mathrm{CLCF}(\mathrm{dB})$ & $\mathrm{FW}(\mathrm{dB})$ \\
\hline 3.0 & 2.7 & 0.2 & $0.8,0.6,0.3$ & $0.8,0.5,0.2$ \\
\hline 3.0 & 2.7 & 0.5 & $0.2,0.1,0.1$ & $0.2,0.0,0.3$ \\
\hline 3.0 & 2.7 & 0.9 & $0.1,0.1,0.4$ & $0.2,0.2,0.9$ \\
\hline 3.0 & 3.0 & 0.2 & $2.0,1.2,1.3$ & $2.0,1.1,1.0$ \\
\hline 3.0 & 3.0 & 0.5 & $0.2,0.6,0.6$ & $0.2,0.5,0.1$ \\
\hline 3.0 & 3.0 & 0.9 & $0.7,0.6,1.1$ & $0.7,0.2,0.2$ \\
\hline 3.0 & 3.3 & 0.2 & $2.1,1.9,1.5$ & $2.1,1.7,1.1$ \\
\hline 3.0 & 3.3 & 0.5 & $0.3,0.8,1.3$ & $0.2,0.6,0.6$ \\
\hline 3.0 & 3.3 & 0.9 & $1.2,0.8,1.6$ & $1.2,0.3,0.2$ \\
\hline 3.0 & 3.5 & 0.2 & $2.1,1.6,1.6$ & $2.1,1.3,1.0$ \\
\hline 3.0 & 3.5 & 0.5 & $0.9,1.4,2.0$ & $0.9,1.1,1.1$ \\
\hline 3.0 & 3.5 & 0.9 & $0.7,0.6,1.6$ & $0.7,0.0,0.1$ \\
\hline 4.0 & 2.7 & 0.2 & $0.2,0.5,0.4$ & $0.1,0.4,0.1$ \\
\hline 4.0 & 2.7 & 0.5 & $1.0,0.3,0.1$ & $1.1,0.5,0.5$ \\
\hline 4.0 & 2.7 & 0.9 & $0.5,0.1,0.0$ & $0.4,0.2,0.7$ \\
\hline 4.0 & 3.0 & 0.2 & $1.4,1.3,0.6$ & $1.4,1.1,0.1$ \\
\hline 4.0 & 3.0 & 0.5 & $0.2,0.6,1.3$ & $0.3,0.3,0.6$ \\
\hline 4.0 & 3.0 & 0.9 & $0.2,0.3,1.3$ & $0.3,0.2,0.0$ \\
\hline 4.0 & 3.3 & 0.2 & $1.0,1.6,1.9$ & $1.0,1.3,1.2$ \\
\hline 4.0 & 3.3 & 0.5 & $1.3,1.6,1.8$ & $1.2,1.2,0.7$ \\
\hline 4.0 & 3.3 & 0.9 & $0.8,1.0,2.6$ & $0.7,0.2,0.4$ \\
\hline 4.0 & 3.5 & 0.2 & $1.5,1.8,2.5$ & $1.5,1.4,1.6$ \\
\hline 4.0 & 3.5 & 0.5 & $2.2,1.7,2.6$ & $2.1,1.2,1.3$ \\
\hline 4.0 & 3.5 & 0.9 & $0.4,1.8,3.0$ & $0.3,0.8,0.4$ \\
\hline 6.0 & 2.7 & 0.2 & $0.9,0.8,1.0$ & $0.8,0.4,0.3$ \\
\hline 6.0 & 2.7 & 0.5 & $0.9,0.7,0.5$ & $1.0,0.1,0.7$ \\
\hline 6.0 & 2.7 & 0.9 & $0.1,0.6,2.2$ & $0.1,0.4,0.5$ \\
\hline 6.0 & 3.0 & 0.2 & $2.7,1.6,2.4$ & $2.6,0.9,0.9$ \\
\hline 6.0 & 3.0 & 0.5 & $1.7,1.5,2.7$ & $1.5,0.5,0.3$ \\
\hline 6.0 & 3.0 & 0.9 & $1.0,1.9, *$ & $0.7,0.0,0.0$ \\
\hline 6.0 & 3.3 & 0.2 & $1.3,2.8{ }^{*}$ & $1.1,1.8,1.5$ \\
\hline 6.0 & 3.3 & 0.5 & $0.6,2.3, *$ & $0.3,0.8,1.3$ \\
\hline 6.0 & 3.3 & 0.9 & $0.5, *{ }^{*} *$ & $0.1,0.8,0.7$ \\
\hline 6.0 & 3.5 & 0.2 & $1.8, *{ }^{*} *$ & $1.6,2.0,1.9$ \\
\hline 6.0 & 3.5 & 0.5 & $2.6, *{ }^{*} *$ & $2.3,1.9,1.7$ \\
\hline 6.0 & 3.5 & 0.9 & $1.7,{ }^{*}, *$ & $1.1,0.7,0.7$ \\
\hline
\end{tabular}

As the need for easy-to-use formulas for outage probability is clear, approximations need to be done. Working on the uplink [4], derived the distribution function of a ratio of path-losses with shadowing, which is essential for the evaluation of external interference. For that, authors approximate the hexagonal cell with a disk of same area. Authors of [5] assume perfect power control on the uplink, while neglecting fast fading. On the downlink, Chan and Hanly [6] precisely approximate the distribution of the other-cell interference. They, however, provide formulas that are difficult to handle in practice and do not consider fast fading. Immovilli and Merani [7] take into account both channel effects and make several assumptions in order to obtain simplified formulas. In particular, they approximate interference by its mean value. Outage probability is however an explicit function of all distances from receiver to every interferer. Zorzi [8], proposes a formula essentially valid for packet radio networks rather than for cellular systems. Authors of [9] provide some interesting characterizations and upper bounds of the outage probability but neglects the slowly varying path-gains. In [10], authors consider both shadowing and fast fading but assume a single interferer.

In this paper, we propose two methods to analyze the outage probability of mobile stations located at any distance $r$ from their serving base station (BS). The first method, based on the Fenton-Wilkinson approach [11], approximates a sum of log-normal random variables as a log-normal random variable and approximates fast fading coefficients in interference terms by their average value. We denote it FWBM (for Fenton-Wilkinson Based Method). The second one is based on the central limit theorem for causal functions [12]. It allows to approximate a sum of positive random variables by a gamma distribution. We denote it CLCFM (for central limit theorem for causal functions method). Each method allows to establish a simple and easily computable outage probability formula, which jointly takes into account path-loss, shadowing and fast fading. We compare our proposed formulas with results obtained with extensive Monte Carlo simulations in a classical hexagonal network. At last, we rely on fluid model proposed in $[13,14]$ in order to express the outage probability as a simple analytical expression depending only on the distance to the serving BS. Such an expression allows further integrations much more easily than with existing formulas. Note that part of the presented results are included in [15].

The paper is organized as follows. FWBM is explained in sect. 3 . We derive the outage probability while considering first only path-loss and shadowing and then path-loss, shadowing and fast fading jointly. Sect. 4 develops the CLCF method. Outage probability is calculated while considering first only path-loss and fast fading and then path-loss, shadowing and fast fading jointly. The computation is based on the fluid model (sect. 5). In sect. 6, we validate our approach and compare analytical expressions with results obtained through Monte Carlo simulations. 


\section{Interference model}

We first define the interference model assumed in this paper. We consider a hexagonal cellular network with frequency reuse one and we focus on the downlink. We are interested in evaluating the SIR at a mobile station $u$, served by base station $B S_{0}$ and interfered by $N$ interfering base stations. We assume that mobile stations are attached to their closest BS.

\subsubsection{Propagation Model}

The power received by $u$ depends on the radio channel state and varies with time due to fading effects (shadowing and fast fading). Let $P_{j}$ be the transmission power of base station $j$, the power $p_{j, u}$ received by $u$ can be written as:

$$
p_{j, u}=P_{j} K r_{j, u}^{-\eta} X_{j, u} Y_{j, u} .
$$

The term $P_{j} \mathrm{Kr}_{j, u}^{-\eta}$, where $\mathrm{K}$ is a constant, represents the mean value of the received power at distance $r_{j, u}$ from the transmitter $\left(B S_{j}\right) . X_{j, u}$ is a random variable (RV) representing the Rayleigh fading effects, whose pdf is $p_{X}$ $(x)=e^{-x}$. The term $Y_{j, u}=10^{\xi_{j, u} / 10}$ is a lognormal RV characterizing shadowing. $\xi_{j, u}$ is a Normal distributed RV, with zero mean and standard deviation, $\sigma$, which is typically between 0 and $8 \mathrm{~dB}$. Parameter $\eta$, which is typically between 2.7 and 3.5 , is the path-loss exponent.

\subsubsection{SIR Calculation}

The interference power received by a mobile $u$ can be written as:

$$
p_{\mathrm{ext}, u}=\sum_{j=1}^{N} P_{j} K r_{j, u}^{-\eta} X_{j, u} Y_{j, u} .
$$

We consider in this paper that the thermal noise is negligible (in a urban environment) and so we focus on the SIR rather than on the signal to interference plus noise ratio (SINR). Considering moreover that all BS transmit with the same power $P_{0}$, we can express the SIR expression (dropping index $u$ and setting $r_{0, u}=r$ ):

$$
\gamma=\frac{r^{-\eta} X_{0} Y_{0}}{\sum_{j=1}^{N} r_{j}^{-\eta} X_{j} Y_{j}}
$$

\subsubsection{Outage Probability}

In this paper, we define the outage probability as the probability that the SIR at $u$ falls below a given threshold $\delta$. Note that while varying $\delta$, we obtain the definition of the SIR CDF. In this paper, we indifferently speak of outage probability or CDF.

$$
\mathbb{P}(\gamma<\delta)=\mathbb{P}\left(\frac{r^{-\eta} X_{0} Y_{0}}{\sum_{j=1}^{N} r_{j}^{-\eta} X_{j} Y_{j}}<\delta\right) .
$$

\section{Fenton-Wilkinson based method}

In this section, we propose a first method based on the Fenton-Wilkinson approximation. We first analyze the path-loss and shadowing impact. We then extend the result to the joint influence of path-loss, shadowing and fast fading based on the previous obtained result.

\subsection{Path-loss and shadowing impact}

The power $p_{j}$ received by $u$ can be written in this case:

$$
p_{j}=P_{j} K r_{j}^{-\eta} Y_{j}
$$

The probability density function (PDF) of this slowly varying received power is given by

$$
p_{Y}(s)=\frac{1}{a \sigma s \sqrt{\pi}} \exp \left[-\left(\frac{\ln (s)-a m}{\sqrt{2} a \sigma}\right)^{2}\right]
$$

Where $a=\frac{\ln 10}{10}, m=\frac{1}{a} \ln \left(K P_{j} r_{j}^{-\eta}\right)$ is the (logarithmic) received mean power expressed in decibels $(\mathrm{dB})$, which is related to the path-loss and $\sigma$ is the (logarithmic) standard deviation of the mean received signal due to the shadowing.

The SIR at user $u$ is now given by:

$$
\gamma=\frac{r^{-\eta} Y_{0}}{\sum_{j=1}^{N} r_{j}^{-\eta} Y_{j}} .
$$

We see that the SIR can be written $\gamma=1 / F$ with

$$
F=\frac{\sum_{j=1}^{N} r_{j}^{-\eta} Y_{j}}{r^{-\eta} Y_{0}} \text {. }
$$

The factor $F$ is defined for any mobile $u$ and it is thus location dependent. The numerator of this factor is a sum of log-normally distributed RV, which can be approximated by a log-normally distributed RV [2]. The denominator of the factor is a log-normally distributed RV. $F$ can thus be approximated by a log-normal RV. Using the Fenton-Wilkinson [11] method, we can calculate the logarithmic mean and standard deviation, $m_{f}$ and $s_{f}$ of $F$ for any mobile at the distance $r$ from its serving $\mathrm{BS}, B S_{0}$ (see Appendix 1):

$$
m_{f}=\frac{1}{a} \ln (f(r, \eta) H(r, \sigma)),
$$




$$
s_{f}^{2}=2\left(\sigma^{2}-\frac{1}{a^{2}} \ln H(r, \sigma)\right),
$$

where

$$
\begin{aligned}
& H(r, \sigma)=e^{a^{2} \sigma^{2} / 2}\left(G(r, \eta)\left(e^{a^{2} \sigma^{2}}-1\right)+1\right)^{-\frac{1}{2}}, \\
& G(r, \eta)=\frac{\sum_{j} r_{j}^{-2 \eta}}{\left(\sum_{j} r_{j}^{-\eta}\right)^{2}}, \\
& f(r, \eta)=\frac{\sum_{j} r_{j}^{-\eta}}{r^{-\eta}} .
\end{aligned}
$$

From Eqs. (4) and (9), we notice that $f(r, \eta)$ represents the factor $F$ without shadowing.

The outage probability is now defined as the probability for the SIR $\gamma$ to be lower than a threshold value $\delta$ and can be expressed as:

$$
\begin{aligned}
\mathbb{P}(\gamma<\delta) & =1-\mathbb{P}\left(\frac{1}{\delta}>F\right) \\
& =1-\mathbb{P}\left(10 \log _{10}\left(\frac{1}{\delta}\right)>10 \log _{10}(F)\right) \\
& =Q\left[\frac{10 \log _{10}\left(\frac{1}{\delta}\right)-m_{f}}{s_{f}}\right] .
\end{aligned}
$$

where $Q$ is the complementary function: $Q(u)=\frac{1}{2} \operatorname{er} f c\left(\frac{u}{\sqrt{2}}\right)$.

\subsection{Path-loss, shadowing and fast fading impact}

In this case, the outage probability can be expressed as:

$$
\mathbb{P}(\gamma<\delta)=1-\mathbb{P}\left(r^{-\eta} X_{0} Y_{0}>\delta\left(\sum_{j=1}^{N} r_{j}^{-\eta} X_{j} Y_{j}\right)\right) .
$$

The interference power received by a mobile $u$ due to fast fading effects varies with time. As a consequence, the fast fading can increase or decrease the power received by $u$. We consider that the increase of interfering power due to fast fading coming from some base stations are compensated by the decrease of interfering powers coming from other base stations. As a consequence, for the interfering power, only the slow fading effect has a significant impact on the SIR. We thus assume that $\forall j \neq 0, X_{j}$ $\approx E\left[X_{j}\right]=1$ (this assumption will be validated by simulations in the next section), and we can write $\mathbb{P}\left(r^{-\eta} X_{0} Y_{0}>\delta\left(\sum_{j=1}^{N} r_{j}^{-\eta} X_{j} Y_{j}\right)\right) \approx \mathbb{P}\left(r^{-\eta} X_{0} Y_{0}>\delta\left(\sum_{j=1}^{N} r_{j}^{-\eta} Y_{j}\right)\right)$.
So we have:

$$
\begin{aligned}
\mathbb{P}(\gamma<\delta) & =1-\mathbb{P}\left(X_{0} Y_{0}>\delta \frac{1}{r^{-\eta}} \sum_{j=1}^{N} r_{j}^{-\eta} Y_{j}\right) \\
& =1-\mathbb{P}\left(X_{0}>\delta F\right) \\
& =1-\int_{0}^{\infty} \mathbb{P}(x>\delta F) p_{X}(x) d x \\
& =1-\int_{0}^{\infty} \mathbb{P}\left(10 \log _{10}\left(\frac{x}{\delta}\right)>10 \log _{10}(F)\right) e^{-x} d x .
\end{aligned}
$$

As a consequence, the outage probability for a mobile located at a distance $r$ from its serving BS, taking into account path-loss, shadowing and fast fading can be written as:

$$
\mathbb{P}(\gamma<\delta)=\int_{0}^{\infty} Q\left[\frac{10 \log _{10}\left(\frac{x}{\delta}\right)-m_{f}}{s_{f}}\right] e^{-x} d x
$$

\section{Central limit theorem for causal functions method}

In this section, we adopt a different path for deriving the SIR CDF. We first express the outage probability by considering the path-loss and fast fading. Afterwards, we use this result in order to introduce the shadowing impact.

\subsection{Path-loss and fast fading impact}

Assuming only fast fading channel, the SIR is given by:

$$
\gamma=\frac{r^{-\eta} X_{0}}{\sum_{j=1}^{N} r_{j}^{-\eta} X_{j}}=\frac{S}{I}
$$

where:

$$
S=r^{-\eta} X_{0}, \quad I=\sum_{j=1}^{N} r_{j}^{-\eta} X_{j}
$$

are two independent RV. To calculate the outage probability, we need to calculate first, the probability distribution function (PDF) $f_{S}(x)$ of $S$ and the PDF $f_{I}(x)$ of $I$. The PDF of the useful power is given by [16]:

$$
f_{S}(x)=\frac{1}{r^{-\eta}} e^{-\frac{x}{r^{-\eta}}} .
$$

We now approximate the interference PDF using the central limit theorem for causal functions [12] by a Gamma distribution given by:

$$
f_{I}(\gamma)=\frac{\gamma^{\nu-1}}{\Gamma(v) \lambda^{\nu}} e^{-\frac{\gamma}{\lambda}}
$$


Table 2 CDF difference in dB between Monte Carlo Simulations (SIM) on the one hand and CLCFM and FWBM on the other hand at 5, 50 and $90 \%(\sigma=7$ and 8 dB, * means greater than $3 \mathrm{~dB}$ ).

\begin{tabular}{|c|c|c|c|c|}
\hline$d[\mathrm{Km}]$ & $\eta$ & $\sigma[\mathrm{dB}]$ & CLCF [dB] & $\mathrm{FW}[\mathrm{dB}]$ \\
\hline 7.0 & 2.7 & 0.2 & $0.2,0.9,2.0$ & $0.3,0.1,0.2$ \\
\hline 7.0 & 2.7 & 0.5 & $0.1,1.1,2.8$ & $0.4,0.1,0.1$ \\
\hline 7.0 & 2.7 & 0.9 & $0.1,2.4{ }^{*}{ }^{*}$ & $0.6,0.0,0.1$ \\
\hline 7.0 & 3.0 & 0.2 & $1.5,2.6,{ }^{*}$ & $1.2,1.1,1.0$ \\
\hline 7.0 & 3.0 & 0.5 & $0.8,3.0,{ }^{*}$ & $1.2,0.8,1.2$ \\
\hline 7.0 & 3.0 & 0.9 & $2.4,{ }^{*}, *$ & $1.7,1.0,1.4$ \\
\hline 7.0 & 3.3 & 0.2 & $0.2,{ }^{*},{ }^{*}$ & $0.6,1.3,2.8$ \\
\hline 7.0 & 3.3 & 0.5 & $2.3,{ }^{*}, *$ & $1.7,1.6,2.4$ \\
\hline 7.0 & 3.3 & 0.9 & $2.6,{ }^{*}, *$ & $1.6,1.5,2.0$ \\
\hline 7.0 & 3.5 & 0.2 & $2.9,{ }^{*}, *$ & $2.4,1.8,2.2$ \\
\hline 7.0 & 3.5 & 0.5 & $1.3, *{ }^{*} *$ & $0.6,1.5,2.4$ \\
\hline 7.0 & 3.5 & 0.9 & $1.7, *{ }^{*}{ }^{*}$ & $0.5,2.2,2.7$ \\
\hline 8.0 & 2.7 & 0.2 & $0.4,2.9, *$ & $0.9,0.8,0.6$ \\
\hline 8.0 & 2.7 & 0.5 & $2.6,{ }^{*},{ }^{*}$ & $1.9,0.4,0.3$ \\
\hline 8.0 & 2.7 & 0.9 & $1.0,{ }^{*}, *$ & $0.0,0.4,1.3$ \\
\hline 8.0 & 3.0 & 0.2 & $1.3,{ }^{*}, *$ & $0.6,1.1,1.7$ \\
\hline 8.0 & 3.0 & 0.5 & $2.7,{ }^{*}, *$ & $1.6,1.1,2.2$ \\
\hline 8.0 & 3.0 & 0.9 & $*{ }^{*}, * *$ & $2.0,1.7,2.3$ \\
\hline 8.0 & 3.3 & 0.2 & $2.7,{ }^{*}, *$ & $1.7,2.6,3.0$ \\
\hline 8.0 & 3.3 & 0.5 & $2.1,{ }^{*},{ }^{*}$ & $0.7,1.9,{ }^{*}$ \\
\hline 8.0 & 3.3 & 0.9 & $2.9, *{ }^{*} *$ & $0.5,2.4,{ }^{*}$ \\
\hline 8.0 & 3.5 & 0.2 & $*{ }^{*}, * *$ & $2.1,3.0,{ }^{*}$ \\
\hline 8.0 & 3.5 & 0.5 & $2.7,{ }^{*}, *$ & $1.0,2.2,{ }^{*}$ \\
\hline 8.0 & 3.5 & 0.9 & $*{ }^{*}, * *$ & $1.2,2.2, *$ \\
\hline
\end{tabular}

where $v=\frac{\mathrm{E}[I]^{2}}{\operatorname{var}(I)}$ and $\lambda=\frac{\operatorname{var}(I)}{\mathrm{E}[I]}$. Since $\mathrm{E}\left[X_{j}\right]=1$ and var $\left(X_{j}\right)=1$ for $j=1, \ldots, N$, the mean of the interference power is given by:

$$
\mathrm{E}[I]=\sum_{j=1}^{N} r_{j}^{-\eta} \mathrm{E}\left[X_{j}\right]=\sum_{j=1}^{N} r_{j}^{-\eta} .
$$

The variance of $I$ can be expressed as:

$$
\operatorname{var}(I)=\sum_{j=1}^{N} r_{j}^{-2 \eta} \operatorname{var}\left(X_{j}\right)=\sum_{j=1}^{N} r_{j}^{-2 \eta} .
$$

So we have:

$$
v=\frac{\left(\sum_{j=1}^{N} r_{j}^{-\eta}\right)^{2}}{\sum_{j=1}^{N} r_{j}^{-2 \eta}},
$$

$$
\lambda=\frac{\sum_{j=1}^{N} r_{j}^{-2 \eta}}{\sum_{j=1}^{N} r_{j}^{-\eta}} .
$$

The outage probability can now be derived as follows:

$$
\begin{aligned}
\mathbb{P}(\gamma<\delta) & =\int_{0}^{\infty} \mathbb{P}(S<I \delta \mid I=\gamma) f_{I}(\gamma) d y \\
& =\int_{0}^{\infty} F_{S}(\delta y) f_{I}(y) d y . \\
& =1-\frac{1}{\left(\frac{\lambda}{r^{-\eta}} \delta+1\right)^{v}} .
\end{aligned}
$$

\subsection{Path-loss, shadowing and fast fading impact}

In this section we will consider that the shadowing follows a log-normal distribution. We can again write $\gamma=$ $S / I$ with now

$$
S=r^{-\eta} Y_{0} X_{0}, \quad I=\sum_{j=1}^{N} r_{j}^{-\eta} Y_{j} X_{j} .
$$

We again approximate the interference PDF using the Central Limit Theorem for Causal Functions. We thus need to compute the two quantities $v_{\mathrm{s}}=\frac{\mathrm{E}[I]^{2}}{\operatorname{var}(I)}$ and $\lambda_{\mathrm{s}}=\frac{\operatorname{var}(I)}{\mathrm{E}[I]}$. Since involved RV are independent, the average value of $I$ is given by:

$$
\begin{aligned}
\mathrm{E}[I] & =\sum_{j=1}^{N} r_{j}^{-\eta} \mathrm{E}\left[Y_{j}\right] \mathrm{E}\left[X_{j}\right], \\
& =e^{\frac{a^{2} \sigma^{2}}{2}} \sum_{j=1}^{N} r_{j}^{-\eta} .
\end{aligned}
$$

In the same way, the variance of $I$ is given by:

$$
\begin{aligned}
\operatorname{var}(I) & =\sum_{j=1}^{N} r_{j}^{-2 \eta}\left(\mathrm{E}\left[Y_{j}^{2}\right] \mathrm{E}\left[X_{j}^{2}\right]-\mathrm{E}\left[Y_{j}\right]^{2} \mathrm{E}\left[X_{j}\right]^{2}\right), \\
& =\left(2 e^{2 a^{2} \sigma^{2}}-e^{a^{2} \sigma^{2}}\right) \sum_{j=1}^{N} r_{j}^{-2 \eta} .
\end{aligned}
$$

The two parameters $v_{s}$ and $\lambda_{s}$ can now be obtained:

$$
\begin{aligned}
& v_{s}=\frac{1}{2 e^{a^{2} \sigma^{2}}-1} \frac{\left(\sum_{j=1}^{N} r_{j}^{-\eta}\right)^{2}}{\sum_{j=1}^{N} r_{j}^{-2 \eta}}, \\
& \lambda_{s}=e^{\frac{a^{2} \sigma^{2}}{2}}\left(2 e^{a^{2} \sigma^{2}}-1\right) \frac{\sum_{j=1}^{N} r_{j}^{-2 \eta}}{\sum_{j=1}^{N} r_{j}^{-\eta}} .
\end{aligned}
$$

Recall that $Y_{0}$ follows a log-normal distribution with logarithmic mean 0 and standard deviation $\sigma$. We can thus 
average the outage probability over the variations of $Y_{0}$ :

$$
\begin{aligned}
\mathbb{P}(\gamma<\delta) & =\int \mathbb{P}\left(\gamma<\delta \mid Y_{0}\right) p_{Y}(\gamma) d y \\
& =\int \mathbb{P}\left(r^{-\eta} X_{0}<\frac{\delta}{y} I\right) p_{Y}(\gamma) d y \\
& =1-\int_{0}^{\infty} \frac{1}{\left(\frac{\lambda_{s}}{r^{-n}} \frac{\delta}{\gamma}+1\right)^{v_{s}}} \times \frac{1}{a y \sigma \sqrt{2 \pi}} \exp \left(-\frac{\ln (\gamma)^{2}}{2 a^{2} \sigma^{2}}\right) d y
\end{aligned}
$$

\section{Analytical fluid model}

With the two proposed methods, we obtain expressions of the SIR CDF at a given distance $r$ from the serving base-station. We see however that expressions depend also on the distances $r_{i}$ between the considered mobile terminal and all interfering BS. With FWBM, parameters $m_{f}$ and $s_{f}$ in (11) depend on the $r_{i}$ (see Eqs. (5) and (6)). With CLCFM, parameters $v_{s}$ and $\lambda_{s}$ in (17) depend also on the $r_{i}$ (see Eqs. (15) and (16)). The presence of all distances $r_{i}$ make proposed formulas sometimes uneasy to use for dimensioning purposes. In this section, we thus express the parameters $m_{f}, s_{f}, v_{s}$ and $\lambda_{s}$ as functions dependent only on the distance $r$ using the fluid model.

The fluid model approach has been developed, e.g., in [17]. It consists in replacing on the downlink a given fixed finite number of transmitters (base stations) by an equivalent continuum of transmitters which are distributed according to some distribution function. For a homogeneous and regular cellular network, inteferers are now characterized by the interfering BS density $\rho_{\mathrm{BS}}$. Let denote:

$$
g(\eta)=\sum_{j=1}^{N} r_{j}^{-\eta}
$$

Assuming an infinite network, the fluid model allows us to approximate $g$ by the following function [17] (see Appendix 2 for more details). Introducing the dependence of $g$ on $r$, we obtain:

$$
g(r, \eta)=\frac{2 \pi \rho_{B S}}{\eta-2}\left(2 R_{c}-r\right)^{2-\eta}
$$

where $R_{c}$ is the half inter-BS distance.

In the FWBM method, parameters $f(r, \eta)$ and $G(r, \eta)$ given by Eqs. (9) and (8), respectively, can be expressed as follows:

$$
\begin{aligned}
& f(r, \eta)=\frac{g(r, \eta)}{r^{-\eta}} \\
& G(r, \eta)=\frac{g(r, 2 \eta)}{g(r, \eta)^{2}} .
\end{aligned}
$$

Parameters $m_{f}$ and $s_{f}$ can thus be written as functions only on the distance $r$ to the serving BS. In the same way, for the CLCFM, we have:

$$
\begin{aligned}
& v_{s}=\frac{1}{2 e^{a^{2} \sigma^{2}}-1} \frac{g(r, \eta)^{2}}{g(r, 2 \eta)}, \\
& \lambda_{s}=e^{\frac{a^{2} \sigma^{2}}{2}}\left(2 e^{a^{2} \sigma^{2}}-1\right) \frac{g(r, 2 \eta)}{g(r, \eta)} .
\end{aligned}
$$

\section{Performance evaluation}

In this section, we compare the figures obtained with analytical expressions (11) and (17) to those obtained by Monte Carlo simulations. It is clear that several approximations have been done in order to obtain easy-to-use closed-form formulas: (1) the Fenton-Wilkinson method is known to be accurate for low standard deviations; (2) the Central Limit Theorem for Causal Functions is an approximation; (3) the fluid model also. It is thus important to know to what extent approximations are acceptable.

\subsection{Monte Carlo simulator}

The simulator assumes an homogeneous hexagonal network as the one shown in Figure 1, made of fifteen rings around a central cell. The cell range is denoted $R$, the half-distance between BS is set to $R_{c}=1 \mathrm{~km}$.

The simulation consists in computing at each snapshot the SIR for a uniformly random location in the central cell. This computation can be done independently of the BS output power because noise is supposed to be negligible both in simulations and analytical study. At each snapshot, shadowing (log-normal distribution with standard deviation $\sigma$ ) and fast fading (exponential distribution of mean 1) RV are independently drawn between the MS and the serving BS and between MS and interfering BS. We do not consider correlation

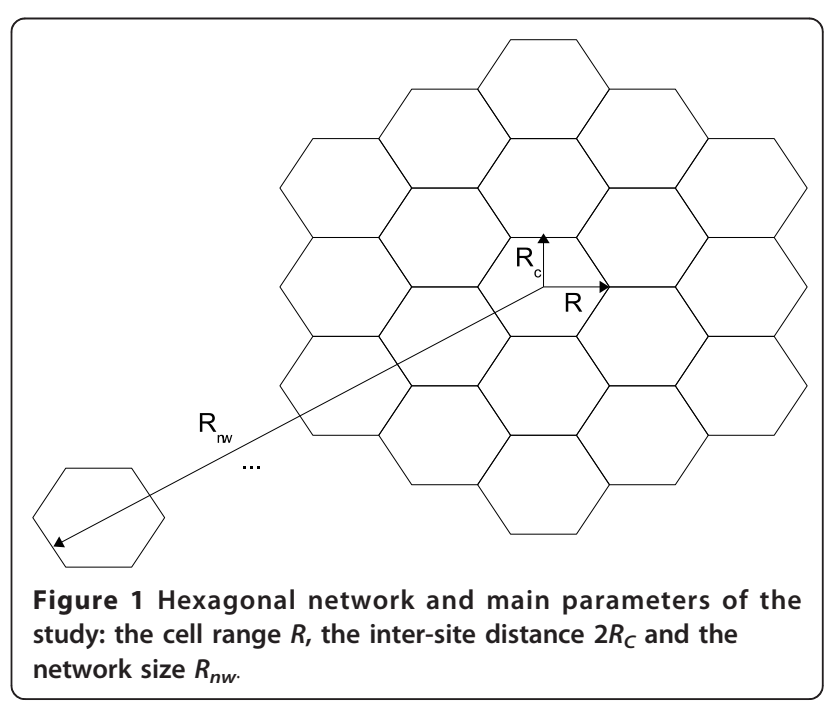


between shadowing coefficient. SIR samples at a given distance from the central BS are recorded in order to compute the outage probability. Five thousand $(5,000)$ snapshots are considered.

\subsection{Results}

In this section, we compare obtained formulas with results obtained by Monte Carlo simulations in a hexagonal network. We study the robustness of our approaches while varying three important parameters: $\sigma$, the standard deviation of the shadowing, $\eta$, the pathloss exponent, and $r$, the distance to the serving BS.

Moreover, we compare the results for the following approaches:

- SIM: results obtained from Monte Carlo simulations;

- FWBM: the Fenton-Wilkinson Based Method in conjunction with the fluid model;

- CLCFM: the Central Limit Theorem for Causal Functions Method in conjunction with the fluid model;

In Figure 2, we compare SIR CDF obtained with SIM, FWBM and CLCFM at $r=0.2 \mathrm{Km}$, for $\eta=3.0$ and while varying $\sigma$ from 3 to $8 \mathrm{~dB}$. Parameter $\sigma$ is definitely the coefficient that influences the most the difference between analysis and simulations. It is clear that the highest is $\sigma$, the highest is the error induced by approximations. For $\sigma=8 \mathrm{~dB}$, the CLCFM is not valid anymore if we consider the whole $\mathrm{CDF}$, but remains accurate for low SIR region. For $\sigma=3 \mathrm{~dB}$, both methods provide very accurate results.

In Figure 3, we study the influence of the distance to the serving BS for $\sigma=4 \mathrm{~dB}$ and $\eta=3.0$. This distance has a small influence on the accuracy of the proposed methods, all analytical CDF fit well with the CDF obtained by simulations.

In Figure 4, we study the path-loss exponent $\eta$ with fixed $\sigma=4 \mathrm{~dB}$ and distance $r=0.2 \mathrm{~km}$. Here again, the parameter has a small influence on the globally good accuracy of the methods. Error seems, however, to increase with $\eta$.

For the sake of completeness, we present in Tables 1 and 2 extensive results for the comparison of SIM, FWBM, and CLCFM. We set three probability thresholds $(5,50$, and $90 \%)$ and we obtain the corresponding SIR thresholds in $\mathrm{dB}$ from the different CDF. Reported figures represent the difference in $\mathrm{dB}$ between the threshold obtained with SIM on the one side and the threshold obtained with FWBM or CLCFM on the other side. Excessive differences (more than $3 \mathrm{~dB}$ ) are marked with a star. From these tables, we can draw some conclusions:

- CLCFM provides accurate results for $\sigma \leq 6 \mathrm{~dB}$ and $\eta \leq 3.0$. For all $\sigma \leq 8 \mathrm{~dB}$, results are still accurate in the low SIR region; this is an interesting result for
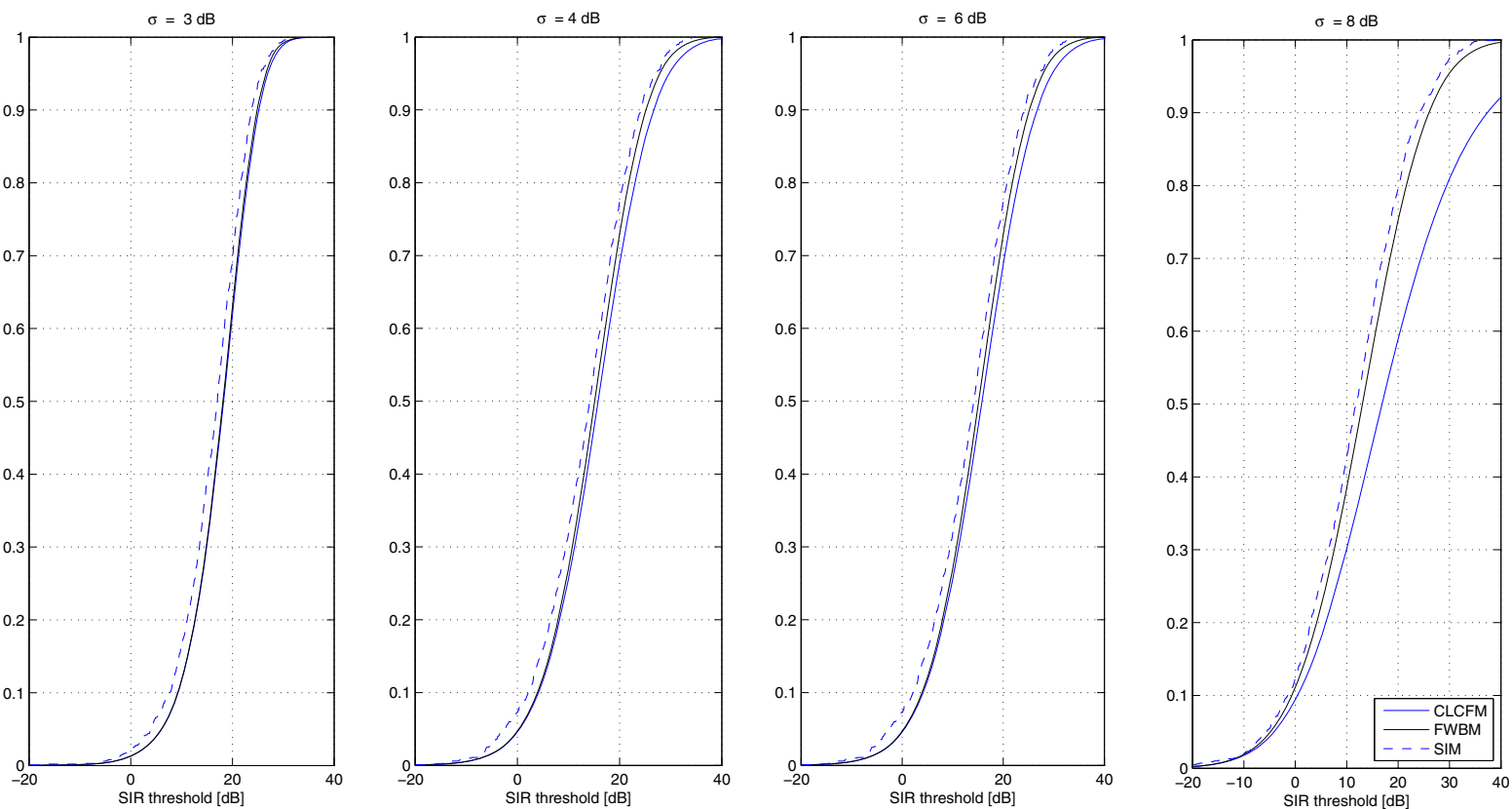

Figure 2 Influence of the shadowing standard deviation $\sigma(\mathrm{dB})$ with $r=0.2 \mathrm{~km}$ and $\eta=3.0$. 

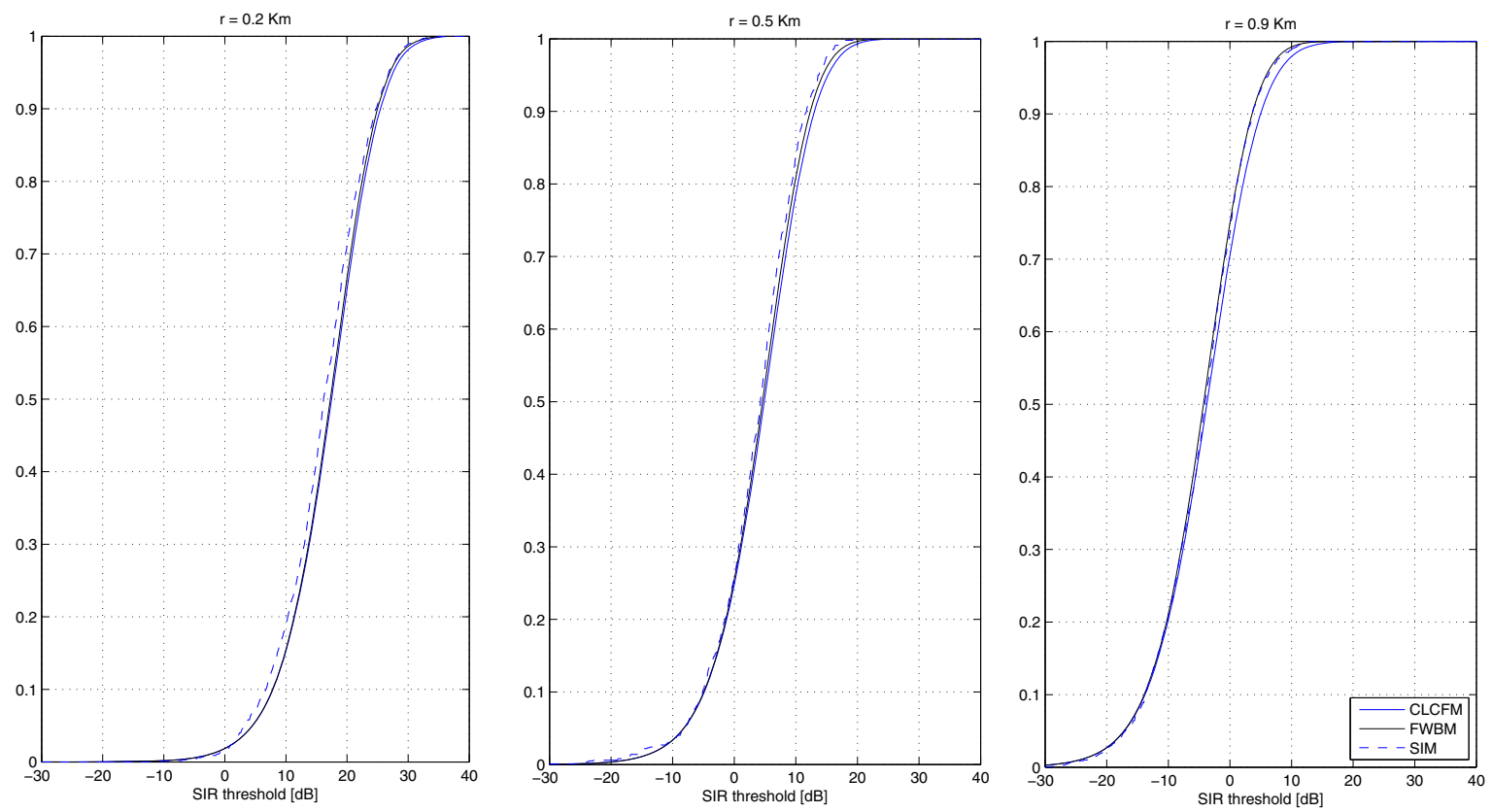

Figure 3 Influence of the distance to the serving BS $r[\mathrm{~km}]$ with $\sigma=4 \mathrm{~dB}$ and $\eta=3.0$.

coverage issues where outage computations are involved.

- FWBM provides accurate results for $\sigma \leq 8 \mathrm{~dB}$ and $\eta \leq 3.0, \eta$ can be greater if $\sigma$ is strictly less than 8 dB. At $\sigma=8 \mathrm{~dB}$ and for $\eta \leq 3.5$, results are still accurate in the low SIR region.

Theses results show that proposed methods, especially FWBM, can provide accurate results for typical values of parameters $r, \sigma$ and $\eta$ usually considered in cellular networks.

\section{Conclusion}

In this paper, we establish simple formulas of the outage probability in cellular networks, while considering pathloss, shadowing and fast fading. Using FWBM approach, we take into account path-loss and shadowing to first express the inverse of the SIR of a mobile located at a given distance of its serving BS as a log-normal random variable. We then consider both path-loss, shadowing and fast fading and give an analytical expression of the outage probability at a given distance of the serving BS. Using CLCFM approach, we take into account path-loss and fast fading to express the SIR of a mobile located at a given distance of its serving BS. We then consider path-loss, fast fading and shadowing and give an analytical expression of the outage probability at a given distance of the serving BS. The fluid model allows us to obtain formulas that only depend on the distance to the serving BS. The analytical model that we propose is validated by comparisons with Monte Carlo simulations. The formulas derived in this paper allow to obtain performances results instantaneously.

The proposed framework is a powerful tool to study performances of cellular networks and to design fine algorithms taking into account the distance to the serving $B S$, shadowing and fast fading. It can particularly easily be used to study frequency reuse schemes in OFDMA systems.

\section{Appendix 1}

In this section, we derive the logarithmic mean and standard deviation of $F$ (see equation (4)) using the Fenton-Wilkinson method. Several classical techniques exist in the literature in order to approximate a sum of lognormal RV, e.g., Schwartz-Yeh [18] and Farley [19]. The former needs complex recursive calculations, the latter assumes identical mean and standard deviation. On the contrary, the Fenton-Wilkinson method provides a closed-form formula for non identical distributed lognormal RV.

Each term of the sum in the numerator of $F$ is a RV following a log-normal distribution. We can write $\ln \left(r_{j}^{-\eta} Y_{j}\right) \propto \mathcal{N}\left(a m_{j}, a^{2} \sigma^{2}\right)$, where $a=\ln (10) / 10$ and $m_{j}=\frac{1}{a} \ln \left(r_{j}^{-\eta}\right)$. The sum $S=\sum_{j=1}^{N} r_{j}^{-\eta} Y_{j}$ can itself be 

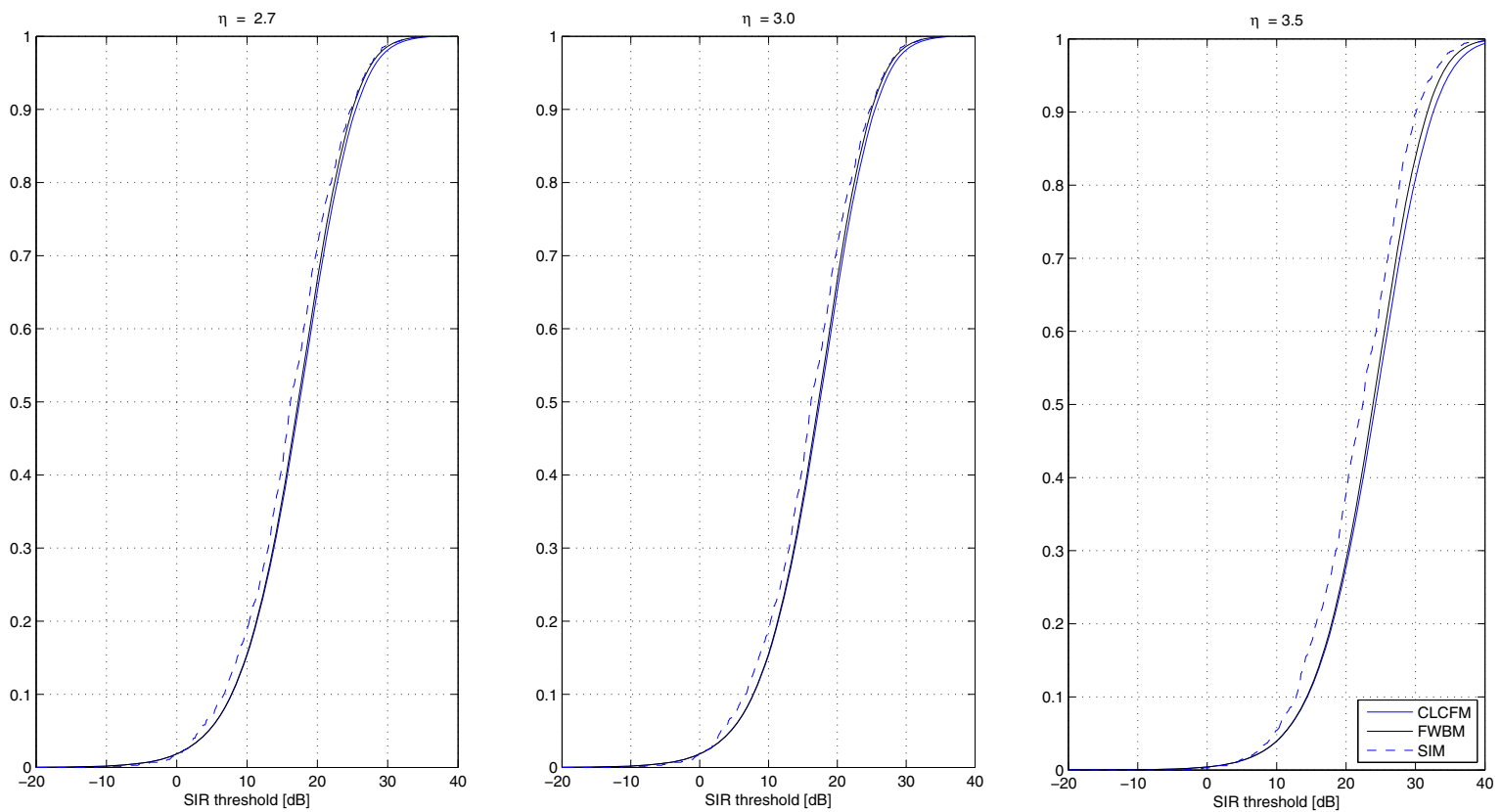

Figure 4 Influence of path-loss exponent $\eta$ with $\sigma=4 \mathrm{~dB}$ and $r=0.2 \mathrm{~km}$.

$$
\begin{aligned}
& \begin{array}{l}
\text { approximated } \\
a m=\ln \left(\sum_{j=1}^{N} e^{a m_{j}}\right)+\frac{a^{2} \sigma^{2}}{2}-\frac{a^{2} \sigma_{s}^{2}}{2},
\end{array} \\
& a^{2} \sigma_{s}^{2}=\ln \left[\left(e^{a^{2} \sigma^{2}}-1\right) \frac{\sum_{j=1}^{N} e^{2 a m_{j}}}{\left(\sum_{j=1}^{N} e^{a m_{j}}\right)^{2}}+1\right] \text {. } \\
& a m=\ln \left(\sum_{j=1}^{N} e^{a m_{j}}\right)+\frac{a^{2} \sigma^{2}}{2}-\frac{a^{2} \sigma_{s}^{2}}{2}, \\
& a^{2} \sigma_{s}^{2}=\ln \left[\left(e^{a^{2} \sigma^{2}}-1\right) \frac{\sum_{j=1}^{N} e^{2 a m_{j}}}{\left(\sum_{j=1}^{N} e^{a m_{j}}\right)^{2}}+1\right] \text {. }
\end{aligned}
$$

These two expressions simplify to:

$$
\begin{aligned}
& a m=\ln \left(\sum_{j=1}^{N} r_{j}^{-\eta}\right)+\frac{a^{2} \sigma^{2}}{2}-\frac{a^{2} \sigma_{s}^{2}}{2}, \\
& a^{2} \sigma_{s}^{2}=\ln \left[\left(e^{a^{2} \sigma^{2}}-1\right) \frac{\sum_{j=1}^{N} r_{j}^{-2 \eta}}{\left(\sum_{j=1}^{N} r_{j}^{-\eta}\right)^{2}}+1\right] .
\end{aligned}
$$

Taking now into account the denominator, the logarithmic mean value of $F$ is simply $a m_{f}=a m-(-\eta \ln (r))$ and the logarithmic standard deviation of $F$ is $a^{2} s_{f}^{2}=a^{2} \sigma_{s}^{2}+a^{2} \sigma^{2}$. The ratio of two log-normal RV is indeed a log-normal RV with mean, the difference of the means, and variance, the sum of the variances. With the definitions of $f, G$ and $H$, we obtain Eqs. (5) and (6). Note that $m_{f}$ and $s_{f}$ are expressed in $\mathrm{dB}$.

\section{Appendix 2}

In this section, we shortly recall the fluid model approach, that will allow us to simplify Eq. 18. The fluid model approach consists of replacing a given fixed finite number of interfering BS by an equivalent continuum of transmitters which are uniformly distributed with density $\rho_{B S}$.

We consider a central cell and a round shaped network around this cell with radius $R_{n w}$. The inter-site distance is $2 R_{c}$ (see Figure 5). Let's consider a mobile $u$ at a distance $r_{u}$ from its serving BS. In the fluid model, each elementary surface $z d z d \theta$ at a distance $z$ from $u$ contains $\rho_{B S} z d z d \theta$ BS which contribute to function $g$. Their contribution to the sum over $j$ is $\rho_{B S} z d z d \theta z^{-\eta}(\eta>2)$. We approximate the integration surface by a ring with centre $u$, inner radius $2 R_{c}-r_{u}$, and outer radius $R_{n w}-r_{u}$ (see Figure 6):

$$
\begin{aligned}
& g\left(r_{u}, \eta\right)=\int_{0}^{2 \pi} \int_{2 R_{c}-r_{u}}^{R_{n w}-r_{u}} \rho_{B S} z^{-\eta} z d z d \theta \\
& =\frac{2 \pi \rho_{B S}}{\eta-2}\left[\left(2 R_{c}-r_{u}\right)^{2-\eta}-\left(R_{n w}-r_{u}\right)^{2-\eta}\right] .
\end{aligned}
$$




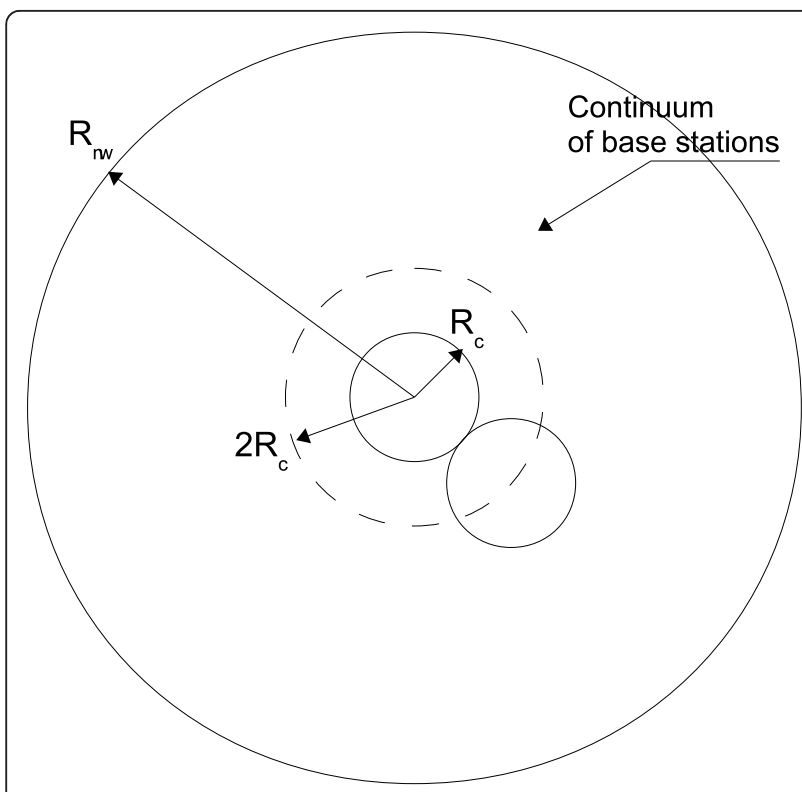

Figure 5 Network and cell of interest in the fluid model; the distance between two BS is $2 R_{c}$ and the network is made of a continuum of base stations.

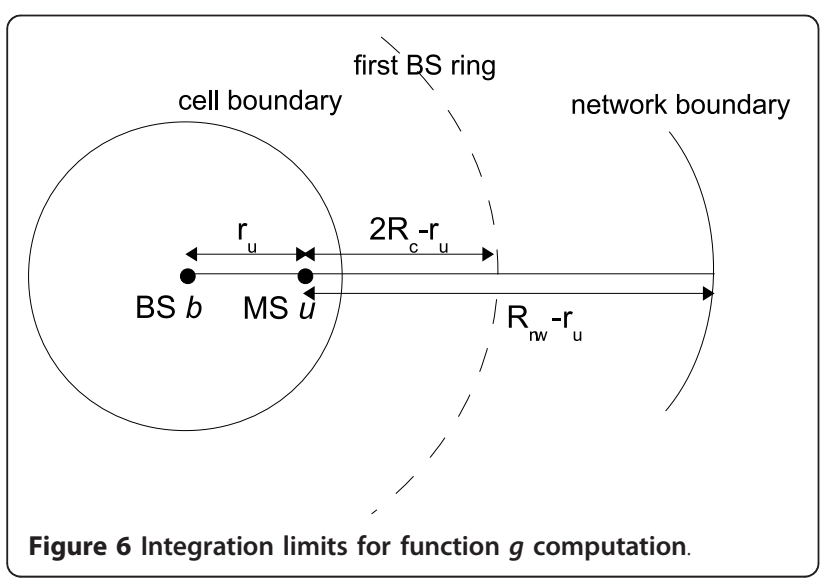

If the network is large, i.e., $R_{n w}$ is big in front of $R_{c}, g$ can be further approximated by (dropping subscript $u$ ):

$$
g(r, \eta)=\frac{2 \pi \rho_{B S}}{\eta-2}\left(2 R_{c}-r\right)^{2-\eta} .
$$

\section{References}

1. M Pratesi, F Santucci, F Graziosi, M Ruggieri, Outage analysis in mobile radio systems with generically correlated log-normal interferers. Commun. IEEE Trans. 48(3), 381-385 (2000). doi:10.1109/26.837041

2. GL Stuber, Principles of Mobile Communications, 2nd Ed. (Kluwer Academic Publishers, Boston/Dordrecht/London, 2001)

3. J-PMG Linnartz, Exact analysis of the outage probability in multiple-user mobile radio. Commun. IEEE Trans. 40(1), $20-23$ (1992). doi:10.1109/ 26.126703

4. JS Evans, D Everitt, Effective bandwidth-based admission control for multiservice cdma cellular networks. Vehi. Technol. IEEE Trans. 48(1), 36-46 (1999). doi:10.1109/25.740058

5. W Veeravalli, A Sendonaris, The coverage-capacity tradeoff in cellular cdma systems. Vehi. Technol. IEEE Trans. 48(5), 1443-1450 (1999). doi:10.1109/ 25.790518

6. CC Chan, SV Hanly, Calculating the outage probability in a cdma network with spatial poisson traffic. Vehi. Technol. IEEE Trans. 50(1), 183-204 (2001). doi:10.1109/25.917918

7. G Immovilli, ML Merani, Simplified evaluation of outage probability for cellular mobile radio systems. Electronics Letters. 27(15), 1365-1367 (1991). doi:10.1049/el:19910859

8. M Zorzi, S Pupolin, Outage probability in multiple access packet radio networks in the presence of fading. Vehi. Technol. IEEE Trans. 43(3), 604-610 (1994). doi:10.1109/25.312811

9. J Papandriopoulos, J Evans, S Dey, Outage-based optimal power control for generalized multiuser fading channels. Commun. IEEE Trans. 54(4), 693-703 (2006)

10. AG Williamson, JD Parsons, Outage probability in a mobile radio system subject to fading and shadowing. Electronics Lett. 21(14), 622-623 (1985). doi:10.1049/el:19850440

11. L Fenton, The sum of log-normal probability distributions in scatter transmission systems. Commun. Sys. IRE Trans. 8(1), 57-67 (1960)

12. A Papoulis, The Fourier Integral and Its Applications, (McGraw-Hill, New York, 1962)

13. J-M Kelif, Admission control on fluid cdma networks, in Modeling and Optimization in Mobile, in Ad Hoc and Wireless Networks, 2006 4th International Symposium on, pp. 1-7 (Apr. 2006)

14. J-M Kelif, E Alman, Downlink fluid model of cdma networks, in Vehicular Technology Conference, 2005. VTC 2005-Spring. 2005 IEEE 61st. 4, 2264-2268 (May 2005)

15. JM Kelif, Marceau Coupechoux, Joint impact of pathloss shadowing and fast fading - an outage formula for wireless networks (2010) ArXiv, abs/ 1001.1110

16. MK Simon, M-S Alouini, Digital Communication Over Fading Channels, (John Wiley \& Sons, Hoboken, New Jersey, 2005)

17. J-M Kelif, M Coupechoux, P Godlewski, Spatial outage probability for cellular networks, in Global Telecommunications Conference, 2007. GLOBECOM '07. IEEE, pp. 4445-4450 (2007)

18. S Schwartz, YS Yeh, On the distribution function and moments of power sums with log-normal components. Bell Syst. Tech. J. 61, 1441-1462 (1982)

19. NC Beaulieu, AA Abu-Dayya, PJ McLane, Comparison of methods of computing lognormal sum distributions and outages for digital wireless applications, in International Conference on Communications, ICC (May 1994)

doi:10.1186/1687-1499-2011-137

Cite this article as: Ben Cheikh et al:: SIR distribution analysis in cellular networks considering the joint impact of path-loss, shadowing and fast fading. EURASIP Journal on Wireless Communications and Networking 2011 2011:137.

\section{Author details}

'Orange Labs, Issy-les-Moulineaux, France ${ }^{2}$ TELECOM ParisTech \& CNRS LTCI, Paris, France

\section{Competing interests}

The authors declare that they have no competing interest.

Received: 31 March 2011 Accepted: 25 October 2011

Published: 25 October 2011 